\title{
Impact d'un effluent urbain de la ville de Taourirt sur la structure des communautés de macroinvertébrés de l'oued Za (Maroc oriental)
}

\section{The effects of an urban effluent from the city of Taourirt on macroinvertebrate community structure in the Za wadi, (Eastern Morocco)}

\author{
Abdeslem Fagrouch, Ali Berrahou et Hassane El Halouani
}

Volume 24, numéro 2, 2011

Reçu le 30 septembre 2009, accepté le 30 juin 2010

URI : https://id.erudit.org/iderudit/1006104ar

DOI : https://doi.org/10.7202/1006104ar

Aller au sommaire du numéro

Éditeur(s)

Université du Québec - INRS-Eau, Terre et Environnement (INRS-ETE)

ISSN

0992-7158 (imprimé)

1718-8598 (numérique)

Découvrir la revue

Citer cet article

Fagrouch, A., Berrahou, A. \& El Halouani, H. (2011). Impact d'un effluent urbain de la ville de Taourirt sur la structure des communautés de macroinvertébrés de l'oued Za (Maroc oriental). Revue des sciences de l'eau / Journal of Water Science, 24(2), 87-101. https://doi.org/10.7202/1006104ar

\section{Résumé de l'article}

Le présent travail s'insère dans le cadre de l'étude de l'impact des rejets domestiques de la ville de Taourirt, sur la qualité physico-chimique et biologique des eaux courantes de l'oued Za, affluent permanent de la rive droite de la Moulouya. Il reçoit ces rejets sans aucun traitement préalable. Cette étude a porté sur trois stations, une station de référence en amont et les deux autres stations en aval des agglomérations. Les prélèvements quantitatifs des macroinvertébrés sont réalisés à l'aide d'une épuisette de type Surber de $0,4 \mathrm{~mm}$ vide de maille permettant d'échantillonner une surface réduite de $0,025 \mathrm{~m}^{2}$. L'inventaire faunistique révèle une diversité taxonomique de 47 familles avec 64 taxons qui ont été récoltés dans les échantillons quantitatifs. Le suivi spatio-temporel de plusieurs paramètres physico-chimiques et biologiques nous a fourni l'image d'une pollution relativement intense. Cette pollution se traduit par une importante charge minérale et organique à l'aval du rejet et qui diminue au fur et à mesure qu'on s'éloigne du rejet grâce à la dégradation d'une partie de la charge organique ou à sa consommation par les microorganismes, à la sédimentation, au facteur de dilution et par les phénomènes naturels d'autoépuration. Les métriques et les indices biotiques, tels que le pointage Biological Monitoring Working Party (BMWP), le Average Score Per Taxa (ASPT), l'indice de diversité, l'indice biotique (IB), la richesse taxonomique et l'équitabilité, ont différencié le site de référence (Pont de Taourirt) des sites légèrement altérés (Ouled Lefkir) et altérés (Rejet Principal). Ces indices biotiques diminuent avec l'augmentation de la dégradation de la qualité des eaux. Par contre, l'indice de Simpson (D) et l'indice biotique de Hilsenhoff modifié (mHBI) augmentent dans les eaux de mauvaise qualité. L'organisation des communautés aux différentes stations est étroitement liée aux conditions du milieu: régime hydrologique, caractéristiques chimiques, végétation riveraine, conditionnant ainsi la richesse taxonomique et l'abondance des macroinvertébrés. 


\section{IMPACT D'UN EFFLUENT URBAIN DE LA VILLE DE TAOURIRT SUR LA STRUCTURE DES COMMUNAUTÉS DE MACROINVERTÉBRÉS DE L'OUED ZA (MAROC ORIENTAL)}

The effects of an urban effluent from the city of Taourirt on macroinvertebrate community structure in the Za wadi, (Eastern Morocco)

ABDESLEM FAGROUCH ${ }^{\prime *}$, ALI BERRAHOU ${ }^{\prime}$, HASSANE EL HALOUANI

${ }^{1}$ Université Mohamed Premier, Faculté des Sciences, Département de Biologie, Laboratoire d'Hydrobiologie et d’Écologie Générale, 60000 Oujda, Maroc.

Reçu le 30 septembre 2009, accepté le 30 juin 2010

\section{RÉSUMÉ}

Le présent travail s'insère dans le cadre de l'étude de l'impact des rejets domestiques de la ville de Taourirt, sur la qualité physico-chimique et biologique des eaux courantes de l'oued $\mathrm{Za}$, affluent permanent de la rive droite de la Moulouya. Il reçoit ces rejets sans aucun traitement préalable. Cette étude a porté sur trois stations, une station de référence en amont et les deux autres stations en aval des agglomérations. Les prélèvements quantitatifs des macroinvertébrés sont réalisés à l'aide d'une épuisette de type Surber de $0,4 \mathrm{~mm}$ vide de maille permettant d'échantillonner une surface réduite de $0,025 \mathrm{~m}^{2}$. L'inventaire faunistique révèle une diversité taxonomique de 47 familles avec 64 taxons qui ont été récoltés dans les échantillons quantitatifs. Le suivi spatio-temporel de plusieurs paramètres physico-chimiques et biologiques nous a fourni l'image d'une pollution relativement intense. Cette pollution se traduit par une importante charge minérale et organique à l'aval du rejet et qui diminue au fur et à mesure qu'on s'éloigne du rejet grâce à la dégradation d'une partie de la charge organique ou à sa consommation par les microorganismes, à la sédimentation, au facteur de dilution et par les phénomènes naturels d'autoépuration. Les métriques et les indices biotiques, tels que le pointage Biological Monitoring Working Party (BMWP), le Average Score Per Taxa (ASPT), l'indice de diversité, l'indice biotique (IB), la richesse taxonomique et l'équitabilité, ont différencié le site de référence (Pont de Taourirt) des sites légèrement altérés (Ouled Lefkir) et altérés (Rejet Principal). Ces indices biotiques diminuent avec l'augmentation de la dégradation de la qualité des eaux. Par contre, l'indice de Simpson (D) et l'indice biotique de Hilsenhoff modifié (mHBI) augmentent dans les eaux de mauvaise qualité. L'organisation des communautés aux différentes stations est étroitement liée aux conditions du milieu : régime hydrologique, caractéristiques chimiques, végétation riveraine, conditionnant ainsi la richesse taxonomique et l'abondance des macroinvertébrés.

Mots clés : Eaux courantes, impacts anthropiques, macroinvertébrés, métriques, indices biologiques, oued Za, Maroc oriental. 


\section{ABSTRACT}

The present work fits in the framework of a survey of the impact of domestic discharges from the city of Taourirt on the physicochemical and biological quality of the running waters of the $\mathrm{Za}$ wadi, which is a permanent tributary of the Moulouya River. The domestic releases do not receive any treatment before their discharge. The study was carried out at three stations: a reference station upstream from the discharge point and the two downstream stations. The quantitative collection of the macroinvertebrates was performed with a Surber net $(0.4 \mathrm{~mm}$ aperture mesh) that allowed sampling of a reduced surface area of $0.025 \mathrm{~m}^{2}$. The inventory of the benthic fauna revealed a taxonomic diversity of 47 families and 64 taxa harvested in the quantitative samples. The spatiotemporal monitoring of several physicochemical and biological variables provided evidence of a relatively intense pollution. This pollution resulted in an important inorganic and organic load to the wadi downstream from the discharge point. This load decreased downstream as a result of the degradation of part of the organic load, due to the action of heterotrophic micro-organisms, and as a result of sedimentation, dilution and natural self-purification phenomena. Use of various metrics and biotic indices (e.g., Biological Monitoring Working Party (BMWP) score, Average Score Per Taxa (ASPT), Shannon-Wiever Diversity Index $(\mathrm{H})$, Biotic Index (IB), taxonomic richness and equitability) differentiated the reference site (Bridge of Taourirt) from the slightly affected (Ouled Lefkir) and deteriorated (Principal Discharge) sites. These biotic indices decrease with increased degradation of water quality. On the other hand, the Simpson index (D) and the Modified Hilsenhoff Biotic Index (mHBI) increase in poor water quality. The organization of the communities at the different sampling sites appeared to be closely linked to the environmental conditions: hydrological mode, chemical characteristics and riparian vegetation controlled the taxonomic richness and abundance of the macroinvertebrates.

Key words: Running Water, Anthropogenic Impacts, Macroinvertebrates, Metrics, Biological Indices, Za Wadi, Eastern Morocco.

\section{INTRODUCTION}

La population de Taourirt, estimée à 206580 personnes en 2004, provoque une pression énorme sur les ressources en eau. Cette ville située sur le bassin versant de l'oued $\mathrm{Za}$ jouit d'un climat méditerranéen maghrébin de type semi-aride. Les activités anthropiques dans la région telles que l'agriculture et le rejet des eaux usées contribuent à la dégradation de la qualité de l'eau. Au Maroc, les basses eaux, surtout en été, limitent la capacité d'autoépuration des cours d'eau, ce qui les rend vulnérables à la pollution.

Les communautés aquatiques sont les premiers éléments affectés par les modifications physiques et chimiques des écosystèmes. Dans le "Rapid Bioassessment Protocol ", BARBOUR et al. (1999) recommandent leur utilisation pour le suivi de l'intégrité écologique des eaux de surface. Les systèmes les plus connus sont le "River Invertebrate Prediction and Classification System "(WRIGHT et al., 1993) et ses dérivés, l'» Australian River Assessment System » (PARSONS et NORRIS, 1996), le "Benthic Assessment of Sediment " (REYNOLDSON et al., 1995) et les indices multimétriques américains (BARBOUR et al., 1995, 1999). De nombreuses méthodes ont été proposées pour déterminer la qualité biologique de l'eau (HELLAWELL, 1986). En particulier, les méthodes basées sur les communautés des macroinvertébrés benthiques sont devenues de plus en plus populaires (BARBOUR et al., 1996; BARBOUR et al., 1999; BUFFAGNI et al., 2000; DE PAUW et VANHOOREN, 1983; LORENZ et al., 2004; NEDEAU et al., 2003; RESH et JACKSON, 1993; SOLIMINI et al., 2008; THORNE et WILLIAMS, 1997). Si les conditions de la perturbation sont présentes pour une période de temps suffisante pour induire des effets nuisibles au niveau de la population, il devient possible d'évaluer des changements dans la structure de la communauté en utilisant des mesures telles que les indices de diversité et de la structure de la communauté (BUSTOS-BAEZ et FRID, 2003; NORRIS et GEORGES, 1993).

Le déversement dans les rivières d'eaux usées domestiques à des degrés divers de transformation peut conduire à des modifications qualitatives et quantitatives importantes des biotopes dans certains endroits. Les eaux usées non traitées renferment des concentrations élevées d'éléments nutritifs (surtout azote et phosphore), de carbone et une grande quantité de bactéries. Elles contiennent également des matières solides en suspension. Ce sont évidemment les écosystèmes aquatiques et leurs peuplements (invertébrés, poissons,) qui sont les plus touchés par les rejets.

La présente étude visait à apprécier l'impact des eaux usées de la ville de Taourirt sur la biodiversité en macroinvertébrés de l'oued $\mathrm{Za}$ et à tester la capacité de métriques et d'indices biotiques dans l'évaluation de l'intégrité biologique de l'oued ZA. 


\section{SITE D'ÉTUDE, MATÉRIELS ET METHODES}

\subsection{Description des stations}

L'oued Za, situé au Maroc Oriental, est un affluent permanent de la rive droite de la Moulouya (Figure 1). Son bassin versant à une superficie de $18000 \mathrm{~km}^{2}$. Il prend sa source dans les Hauts-Plateaux orientaux. Il draine la partie méridionale et occidentale de la chaîne des Horsts, formée de dolomies et de calcaires plus au moins marneux. Sa direction générale est sud-est nord-ouest. Il rejoint la Moulouya à Melga El Ouidane, à l'aval de la ville de Taourirt. Il traverse d'amont en aval l'étage bioclimatique méditerranéen aride dans les Hauts-Plateaux, semi-aride dans la chaîne des Horsts et aride dans la région de Taourirt (BERRAHOU et al., 2001b).
Compte tenu de l'objectif général de l'étude, trois stations sont retenues sur le cours inférieur de l'oued $\mathrm{Za}$ :

- Taourirt au niveau du pont (PT) : située en amont des agglomérations, altitude : $350 \mathrm{~m}$; profondeur maximale : $20 \mathrm{~cm}$; largeur: $20 \mathrm{~m}$. Le substrat est hétérogène, constitué de blocs, de galets, de graviers, de sable et limons. La végétation des rives est composée de quelques pieds de roseaux. À quelque mètres en aval de cette station une grande quantité d'eau est détournée pour l'irrigation.

- Rejet principal (RP) : altitude : $280 \mathrm{~m}$; profondeur maximale : $25 \mathrm{~cm}$; largeur : $15 \mathrm{~m}$. C'est le point de déversement des eaux usées d'origine domestique de la ville de Taourirt dans l'oued $\mathrm{Za}$. Le fond est constitué de quelques blocs, de galets, de sable sur les rives et de boues des eaux usées et de macro et microdéchets. Dans le lit de l'oued sur la rive droite de cette station se trouve une terrasse d'alluvions de quelques mètres

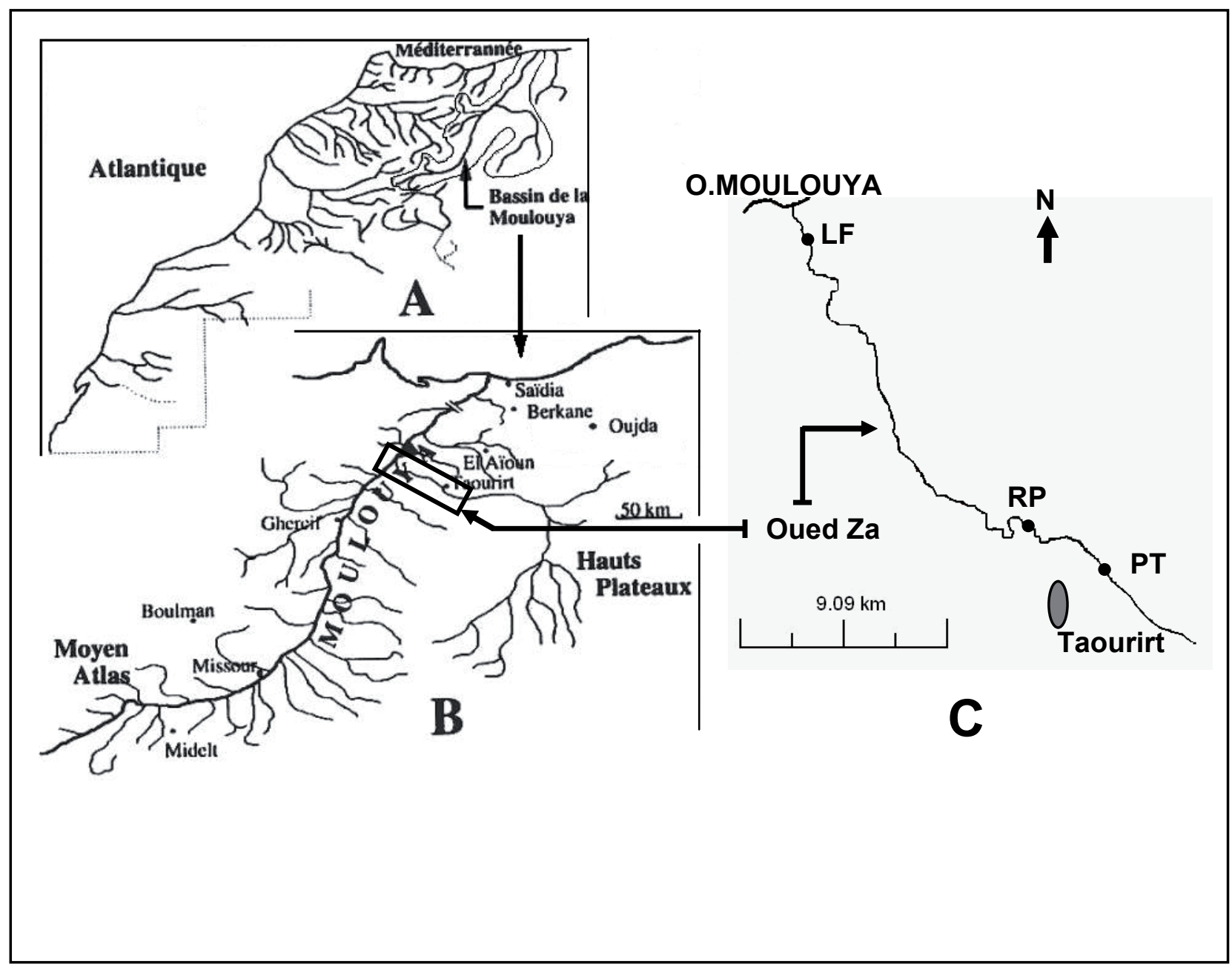

Figure 1. Situation géographique des stations d'étude. A : Position de la Moulouya dans le réseau hydrographique du Maroc. B : Réseau hydrographique de la Moulouya. C : Oued Za. PT : Pont de Taourirt. RP : Rejet Principal. LF : Ouled Lefkir. Geographical location of the study stations. A: Position of the Moulouy a River in the hydrographical network of Morocco; B: Hydrographical network of the Moulouya River; C: Za wadi; PT: Bridge of Taourirt; RP: Principal Discharge; LF: Ouled Lefkir. 
de large et de quelques centaines de mètres de long occupée par des pieds de Tamarix. Cette station est très eutrophisée.

- Ouled Lefkir (LF) : située en aval des agglomérations; altitude : $230 \mathrm{~m}$; profondeur maximale : $30 \mathrm{~cm}$; largeur : $10 \mathrm{~m}$. La plaine est mois étalée sur la rive droite que sur la rive gauche. Les clôtures des champs sont formées d'arbres d'olivier et les cultures y sont variées. Le substrat est formé de blocs semi-emmergie, de pierres, de galets de différentes tailles, de graviers et de sable sur les bords. La végétation se limite à quelques pieds de Nerium et Tamarix sur la rive gauche, et quelques pieds de roseaux sur la rive droite. Sur les bords et sur le fond, il y a dépôt de sédiments.

\subsection{Techniques de prélèvement}

La technique d'échantillonnage des macroinvetébrés benthiques généralement utilisée dans les cours d'eau marocains est celle des épuisettes de type Surber (BERRAHOU et al., 2000). L'épuisette utilisée a $0,4 \mathrm{~mm}$ de vide de maille et permet d'échantillonner une surface de prélèvement de $0,025 \mathrm{~m}^{2}$ $(25 \times 20 \mathrm{~cm})$. Le nombre de prélèvements est de l'ordre de six par station, effectués sur des habitats différents (trois habitats lotiques et trois lentiques). Les prélèvements ont été réalisés chaque mois depuis juin 2004 jusqu'à novembre 2004. Les échantillons sont conservés dans le formol à $10 \%$ sur le terrain. Ils sont triés au laboratoire sous loupe binoculaire. Les macroinvertébrés ont été identifiés au niveau de détermination le plus précis possible.

\subsection{Paramètres analysés et méthodes de dosage utilisées}

Les paramètres physico-chimiques globaux $\left(\mathrm{T}^{\circ}, \mathrm{pH}, \mathrm{O}_{2}\right.$ dissous, conductivité) ont été mesurés sur le terrain durant la période d'étude. Les méthodes d'analyse des descripteurs physiques et chimiques sont résumées dans le tableau 1.

\subsection{Régime de débit}

Les mesures des débits des trois stations étudiées ont été effectuées sur le terrain pour chaque mois pendant toute la période d'étude. Ces mesures ont été réalisées de la façon suivante :

- L'estimation de la vitesse du courant en utilisant un flotteur et un chronomètre. Le flotteur est posé dans le courant au milieu du cours d'eau et une mesure de temps t est prise pour parcourir une distance (d). La vitesse du courant est donc :

$$
\mathrm{V}=\mathrm{d} / \mathrm{t}\left(\mathrm{m} \bullet \mathrm{s}^{-1}\right)
$$

Tableau 1. Méthodes d'analyses physicochimiques. Table1. Methods used for the physico-chemical analyses.

\begin{tabular}{|c|c|}
\hline Variables & Méthodes \\
\hline $\mathrm{pH}$ & pH-mètre de type WTW HI 991003 \\
\hline Conductivité $\left(\mu \varsigma^{\bullet} \mathrm{cm}^{-1}\right)$ à $20^{\circ} \mathrm{C}$ & Conductimètre de type WTW LF 330 \\
\hline Matières en suspension $\left(\mathrm{mg} \bullet \mathrm{L}^{-1}\right)$ & $\begin{array}{l}\text { Filtration sur membrane millipore }(0,45 \mu \mathrm{m}) \text {, passage à l'étuve et } \\
\text { pesées (AFNOR NFT90-105) }\end{array}$ \\
\hline Demande chimique en oxygène $\left(\mathrm{mg} \mathrm{O}_{2} \bullet \mathrm{L}^{-1}\right)$ & Oxydabilité au bichromate de potassium (AFNOR T90-101) \\
\hline Demande biochimique en oxygène $\left(\mathrm{mg} \mathrm{O}_{2} \bullet \mathrm{L}^{-1}\right)$ & Méthode respirométrique T90 103 d'AFNOR \\
\hline Oxygène dissous $\left(\mathrm{mg} \mathrm{O}_{2} \bullet \mathrm{L}^{-1}\right)$ & Oxymètre de type WTW Oxi 315i/SET \\
\hline 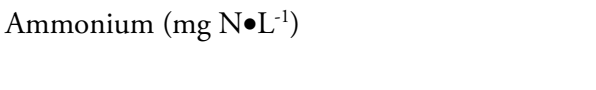 & $\begin{array}{l}\text { AFNOR T90-015 sur un échantillon filtré, par méthode } \\
\text { colorimétrique au bleu d'indophénol }\end{array}$ \\
\hline Nitrites $\left(\mathrm{mg} \mathrm{N} \bullet \mathrm{L}^{-1}\right)$ & AFNOR, 1986 \\
\hline Nitrates $\left(m g ~ N \bullet L^{-1}\right)$ & AFNOR T90-023 \\
\hline 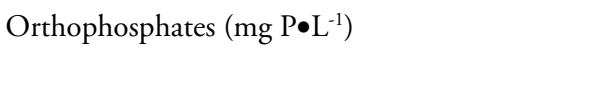 & $\begin{array}{l}\text { AFNOR NF T90-023 sur un échantillon filtré, par méthode } \\
\text { colorimétrique }\end{array}$ \\
\hline
\end{tabular}


- L'estimation de la section mouillée se fait par la mesure de la largeur moyenne $\mathrm{L}$ et de la profondeur moyenne P. On peut calculer la section d'une manière approximative de la façon suivante :

$$
\mathrm{S}=\mathrm{L} \times \mathrm{P}
$$

Le débit est donc calculé par la formule :

$$
\mathrm{Q}=\mathrm{S} \times \mathrm{V}\left(\mathrm{m}^{3} \bullet \mathrm{s}^{-1}\right)
$$

\section{5 les Indices biologiques}

La richesse taxonomique, ou nombre de taxons distincts utilisés dans l'ensemble des indices biologiques, est établie à partir d'un niveau d'identification au genre, à la famille ou plus. Cette catégorie, qui reflète la diversité d'un échantillon (RESH et al., 1995), apparaît comme un bon indicateur de la capacité d'un écosystème à soutenir une variété de taxons.

L'indice de diversité de Shannon-Wiener, qui permet d'apprécier la diversité d'un peuplement, est une quantité positive prenant sa valeur maximale quand un seul élément (espèce par exemple) est présenté en plus d'un exemplaire (individu) et sa valeur minimale quand tous les éléments ont la même fréquence. Les espèces rares ont un poids beaucoup plus faible que les plus communes. Cet indice a pour formule :

$$
\mathrm{H}=-\sum_{\mathrm{i}=1}^{\mathrm{s}}\left(\mathrm{p}_{\mathrm{i}}\right)\left(\log _{2} \mathrm{p}_{\mathrm{i}}\right)
$$

Avec $s=$ nombre total d'espèces de l'échantillon, $\mathrm{p}_{\mathrm{i}}=$ abondance proportionnelle ou pourcentage d'importance de l'espèce se calcule ainsi :

$$
\mathrm{p}_{\mathrm{i}}=\mathrm{n}_{\mathrm{i}} / \mathrm{n}
$$

$\mathrm{n}_{\mathrm{i}}=$ nombre total d'individus de l'espèce de rang $\mathrm{i}$; $\mathrm{n}=$ nombre total d'individus de l'échantillon.

L'indice d'équitabilité ou " everness " (indice de Pielou) correspond au rapport de l'indice de diversité $\mathrm{H}$ sur la valeur maximale de $\mathrm{H}$ en cas d'équirépartition, laquelle correspond au cas où toutes les espèces sont représentées chacune par le même nombre d'individus.

Dans ce cas :

$$
\mathrm{H}_{\max }=\log _{2}(\mathrm{~S})
$$

d'où :

$$
\mathrm{E}=\mathrm{H} / \mathrm{H}_{\max }
$$

où $S=$ le nombre total d'espèces échantillonnées, $\mathrm{H}=$ l'indice de Shannon-Wiener.

L'indice de Simpson (D) permet de mesurer la dominance dans une communauté benthique. Dans notre cas, la dominance calculée est celle de l'échantillon qui est lui-même le reflet de la communauté des macroinvertébrés des sites étudiés. Cet indice est calculé à l'aide de la formule suivante :

$$
\mathrm{D}=1-\sum_{\mathrm{i}=1}^{\mathrm{s}}\left(\mathrm{p}_{\mathrm{i}}\right)^{2}
$$

Avec $S=$ nombre total d'espèces, $\mathrm{p}_{\mathrm{i}}=$ abondance proportionnelle ou pourcentage d'importance de l'espèce se calcule ainsi :

$$
\mathrm{p}_{\mathrm{i}}=\mathrm{n}_{\mathrm{i}} / \mathrm{n}
$$

$\mathrm{p}_{\mathrm{i}}=\mathrm{n}_{\mathrm{i}} / \mathrm{n}$;

$\mathrm{n}_{\mathrm{i}}=$ nombre d'individus d'une espèce dans l'échantillon;

$\mathrm{n}=$ nombre total d'individus de toutes les espèces dans l'échantillon.

La dominance quasi absolue d'une seule espèce est exprimée par un indice proche de 1 . Si les espèces sont nombreuses et ont toutes le même poids dans l'échantillon (équirépartition), l'indice s'approche de 0 .

L'indice biotique (IB) a été déterminé grâce au tableau à double entrée de TUFFERY et VERNEAUX (1968). L'indice biotique ainsi obtenu varie de 0 à 10 . Plus il est élevé, plus les espèces sensibles à la pollution organique sont présentes dans les eaux étudiées et plus le nombre d'unités systématiques est important.

L'indice biotique de Hilsenhoff modifié (mHBI) (Modified Hilsenhoff Biotic Index) : Plusieurs États, y compris le Kentucky, ont employé le mHBI pour évaluer d'autres impacts que l'enrichissement organique et ils ont prouvé que le mHBI est une valeur métrique. Une valeur croissante de mHBI indique la qualité de l'eau décroissante. Cet indice est calculé à l'aide de la formule suivante :

$$
\mathrm{mHBI}=\frac{\sum \mathrm{n}_{\mathrm{i}} \mathrm{xa}_{\mathrm{i}}}{\mathrm{n}}
$$

avec $\mathrm{n}_{\mathrm{i}}=$ nombre d'individus d'une espèce dans l'échantillon;

$\mathrm{a}_{\mathrm{i}}=$ valeur de tolérance d'espèce au rang « $\mathrm{i}$ »;

$\mathrm{n}=$ nombre total d'individus de toutes les espèces dans l'échantillon. 
Le pointage BMWP (Biological Monitoring Working Party) (ARMITAGE et al,. 1983) est utilisé de préférence dans les cours d'eau à faciès lotique. À chaque famille collectée, selon sa tolérance à la pollution (BARBOUR et al., 1999, MANDAVILLE, 2002), correspond un pointage qui est une note de 1 à 10. Le pointage BMWP est la somme des pointages des familles présentes dans l'échantillon. Les pointages varient entre 0 et 250 : plus ils sont élevés, moins le milieu est pollué. À partir de la liste obtenue, il est possible de calculer le pointage BMWP qui est comparé au score obtenu par l'échantillonnage. Un pointage ASPT (Average Score Per Taxon) peut être calculé en divisant le pointage BMWP par le nombre total de taxons récoltés ayant un score (FRIEDRICH et al., 1996). Ce pointage varie entre 0 et 7 .

L'indice d'Éphéméroptères Trichoptères et Odonates (ETO) représente la richesse taxonomique de ces trois groupes (GERRITSEN et al., 1998). L'indice d'ETO égal au nombre total des familles représentées par ces trois groupes dans les échantillons Éphéméroptères, Trichoptères et Odonates sont considérés sensibles à la pollution.

Le pourcentage des dominants : cet indice est une évaluation des taxons les plus dominants (famille ou genre) dans l'échantillon. L'indice est calculé en ajoutant d'abord les deux familles dominantes principales et en le divisant ensuite par le nombre total d'organismes, et en multipliant ce rapport par 100 pour obtenir le pourcentage.

L'indice de racleur/filtreur-collecteur (Rc/FC) est calculé en divisant le nombre total d'individus classifiés comme des racleurs par le nombre total d'individus classifiés en tant que filtreurs-collecteurs dans l'echantillon.

\section{ANALYSES DES DONNÉES}

Vingt-neuf métriques, basées sur la richesse taxonomique, la composition et la tolérance à la pollution, ont été calculées à partir des trois sitesétudiés (Tableau 2). Ces différentes métriques sont couramment utilisées dans des programmes de surveillance biologique (BARBOUR et al., 1999; MANDAVILLE, 2002). Les métriques sont des caractéristiques de l'habitat qui changent d'une façon prévisible avec l'augmentation des perturbations (BARBOUR et al., 1999). Les valeurs de tolérance à la pollution des taxons sont issues des travaux de BARBOUR et al. (1999), BODE et al. (2002) et HILSENHOFF (1988). Dans cette étude nous avons sélectionné les métriques et les indices multimétriques appropriés pour l'évaluation de l'intégrité des écosystèmes aquatiques (BARBOUR et al., 1999; USEPA, 1998, 2000, 2002).

Les valeurs de coefficients corrélations entre les indices biotiques et les paramètres chimiques ont été calculées (Tableau 3) en utilisant le logiciel STATISTICA 6.0, de même pour les différents indices biologiques entre eux (Tableau 4).

\section{RÉSULTATS}

Les résultats des principaux paramètres physico-chimiques et biologiques des trois sites d'étude pour la période allant de juillet 2004 à novembre 2004 sont représentés dans le tableau 2. Les valeurs les plus élevées du débit ont été enregistrées en amont au niveau de la station PT $\left(5,32 \mathrm{~m}^{3} \bullet \mathrm{s}^{-1}\right)$. Par contre, en aval, une légère diminution a été constatée respectivement 3,22 et $3,72 \mathrm{~m}^{3} \bullet \mathrm{s}^{-1}$ pour les stations RP et LF. Ceci est dû à des pompages d'eau à des fins agricoles. En ce qui concerne la vitesse du courant, celle-ci n'est pas homogène tout au long des stations étudiées; elle présente des variabilités d'une station à l'autre avec des valeurs extrêmes allant de $0,832 \mathrm{~m} \bullet \mathrm{s}^{-1}(\mathrm{PT})$ à $1,066 \mathrm{~m} \bullet \mathrm{s}^{-1}(\mathrm{RP})$.

La minéralisation est excessive dans toutes les stations étudiées. La valeur minimale de la conductivité est mesurée au niveau de la station du pont (PT), mais la plus forte minéralisation est marquée au niveau de la station de LF avec $2534 \mu \mathrm{S} \bullet \mathrm{cm}^{-1}$. L'augmentation de la conductivité de l'amont vers l'aval peut s'expliquer en grande partie par la composition minérale des eaux usées domestiques de la ville de Taourirt à laquelle s'ajoute la nature géologique des terrains traversés. BERRAHOU et al. (2001b) ont montré que la conductivité augmente de l'amont vers l'aval de l'oued Moulouya, grâce à la géologie des terrains traversés et aux eaux usées qui se déversent dans la Moulouya.

Les résultats des teneurs en oxygène dissous au niveau des trois stations étudiées rapportent que les stations PT et LF présentent les valeurs élevées avec respectivement $11,27 \mathrm{mg} \mathrm{d} \mathrm{O}_{2} \cdot \mathrm{L}^{-1}$ et $11,49 \mathrm{mg} \mathrm{d} \mathrm{O}_{2} \cdot \mathrm{L}^{-1}$, et présentent ainsi des conditions de saturation et de sursaturation. Cependant, la station RP recevant les rejets d'eaux usées domestiques présente des teneurs plus faibles $\left(7,79 \mathrm{mg} \mathrm{d} \mathrm{d}_{2} \bullet \mathrm{L}^{-1}\right)$ qu'aux stations PT et LF. Cette diminution de la concentration en oxygène dissous peut s'expliquer par l'utilisation de l'oxygène dissous par les microorganismes pour la biodégradation de la matière organique apportée par le rejet domestique. Cependant, au niveau du rejet principal (RP), la diminution de la teneur en oxygène dissous n'est pas significative $\left(7,79 \mathrm{mg} \mathrm{d} \mathrm{d}_{2} \cdot \mathrm{L}^{-1}\right)$. Cette diminution de la concentration en oxygène dissous peut s'expliquer par l'écoulement rapide de l'eau avec un intense 
Tableau 2. Les valeurs moyennes des variables environnementales de six prélèvements (juin 2004-novembre 2004) dans les stations étudiées. PT : Pont de Taourirt; RP : Rejet Principal; LF : Ouled Lefkir.

Table 2. Average values of the environmental variables from six sampling campaigns (June 2004-November 2004) at the studied stations. PT: Bridge of Taourirt; RP: Principal Discharge; LF: Ouled Lefkir.

\begin{tabular}{|c|c|c|c|}
\hline Variables/Stations & PT & $\mathrm{RP}$ & $\mathrm{LF}$ \\
\hline \multicolumn{4}{|l|}{ Physico-chimiques } \\
\hline Altitude (m) & 350 & 280 & 230 \\
\hline Profondeur $(\mathrm{cm})$ & 20 & 25 & 30 \\
\hline Largeur $(\mathrm{m})$ & 20 & 15 & 10 \\
\hline Vitesse $\left(m \bullet s^{-1}\right)$ & 0,832 & 1,066 & 1,035 \\
\hline Débit $\left(\mathrm{m}^{3} \bullet \mathrm{s}^{-1}\right)$ & 5,32 & 3,22 & 3,72 \\
\hline Conductivité $\left(\mu \mathrm{S} \bullet \mathrm{cm}^{-1}\right)$ & 1775 & 1797 & 2490 \\
\hline T eau ${ }^{\circ} \mathrm{C}$ & 22,22 & 22,58 & 23,03 \\
\hline $\mathrm{pH}$ & 7,86 & 8,39 & 7,99 \\
\hline DO (Oxygène Dissous) $\left(\mathrm{mg} \mathrm{L}^{-1}\right)$ & 11,27 & 7,79 & 11,49 \\
\hline $\mathrm{DBO}_{5}\left(\right.$ Demande Biologique en Oxygène) $\left(\mathrm{mg} \bullet \mathrm{L}^{-1}\right)$ & 5,4 & 657,5 & 8 \\
\hline 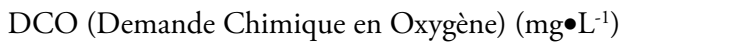 & 11,3 & 1304,5 & 18,4 \\
\hline MES (Matières en Suspension) $\left(\mathrm{mg} \bullet \mathrm{L}^{-1}\right)$ & 56 & 200 & 75 \\
\hline $\mathrm{N}-\mathrm{NH}_{4}($ Ammonium $)\left(\mathrm{mg} \bullet \mathrm{L}^{-1}\right)$ & 0,05 & 1,35 & 0,19 \\
\hline $\mathrm{N}-\mathrm{NO}_{2}($ Nitrites $)\left(\mathrm{mg} \bullet \mathrm{L}^{-1}\right)$ & 0,04 & 0,27 & 0,05 \\
\hline $\mathrm{N}-\mathrm{NO}_{3}($ Nitrates $)\left(\mathrm{mg} \bullet \mathrm{L}^{-1}\right)$ & 1,65 & 10,12 & 3,25 \\
\hline $\mathrm{P}_{-} \mathrm{PO}_{4}$ (Orthophosphates) $\left(\mathrm{mg} \mathrm{L}^{-1}\right)$ & 0,015 & 1,68 & 0,024 \\
\hline \multicolumn{4}{|l|}{ Biologiques } \\
\hline$S$ (richesse taxonomique) & 52 & 16 & 38 \\
\hline H (indice de Shanno-Wiener) & 3,74 & 0,69 & 3,02 \\
\hline Eq (Équitabilité) & 84,06 & 14,73 & 62,41 \\
\hline D (indice de Simpson) & 0,099 & 0,83 & 0,052 \\
\hline IB (Indice Biotique) & 9 & 2,6 & 8,83 \\
\hline mHBI (Modified Hilsenhoff Biotic Index) & 5 & 6,64 & 5,8 \\
\hline BMWP (Biological Monitoring Working Party) & 181 & 23 & 121 \\
\hline ASPT (Average Score Per Taxa) & 3,5 & 1,43 & 3,18 \\
\hline ETO (taxons d'Éphéméroptères, Trichoptères et Odonates) & 22 & 0 & 14 \\
\hline E (taxons d'Éphéméroptères) & 9 & 0 & 5 \\
\hline $\mathrm{T}$ (taxons Trichoptères) & 9 & 0 & 8 \\
\hline O (taxons Odonates) & 4 & 0 & 1 \\
\hline \% ETO (\%d'Éphéméroptères, Trichoptères et Odonates) & 82,0 & 0 & 71,5 \\
\hline \% E (\% Éphéméroptères) & 49,9 & 0 & 48,9 \\
\hline$\% \mathrm{~T}$ (\% Trichoptères) & 31,5 & 0 & 22,5 \\
\hline$\% \mathrm{O}$ (\% Odonates) & 0,5 & 0 & 0,03 \\
\hline \% Di (\% des Diptères) & 13,7 & 104,3 & 12,9 \\
\hline$\%$ Chir (\% Chironomidae) & 10,9 & 103,6 & 10,7 \\
\hline ETO/C (taxons d'ETO/taxons Chironomidae) & 7,5 & 0 & 6,65 \\
\hline$\%$ Dom (\% des 2 familles dominantes) & 42,83 & 103,3 & 49,9 \\
\hline I (taxons d'Intolérants) & 23 & 0 & 7 \\
\hline$\% \operatorname{Tr}(\%$ Tolérants $)$ & 29 & 105 & 46 \\
\hline Cn (taxons des Récupérateurs (Récupérateurs)) & 9 & 0 & 6 \\
\hline \% Cn (\%Récupérateurs) & 6,4 & 0 & 1,9 \\
\hline Rc/FC (taxons des Racleurs/taxons Filtreurs-Collecteurs) & 0,127 & 0,00015 & 0,046 \\
\hline \% FC (\% Filtreurs-Collecteurs) & 29,8 & 90,9 & 24,6 \\
\hline$\%$ Rc (\% Racleurs) & 3,8 & 0,0 & 1,1 \\
\hline NF (Nombre total de Familles) & 37 & 14 & 26 \\
\hline $\mathrm{N} \bullet \mathrm{m}^{-2}$ (Abondance Totale) & 28686 & 48420 & 69933 \\
\hline
\end{tabular}


Tableau 3. Les valeurs de coefficients de corrélations entre les indices biologiques et les variables chimiques les plus appropriés. (Corrélations significatives marquées à $\mathbf{p}<\mathbf{0 , 0 5}$ ).

Table 3. Values of correlation coefficients between the biological indices and the most relevant chemical variables (Significant correlations marked with $p<0,05$ ).

\begin{tabular}{lccccccccc}
\hline Variables & $\mathrm{PH}$ & $\mathrm{DO}$ & $\mathrm{DBO}$ & $\mathrm{DCO}$ & $\mathrm{MES}$ & $\mathrm{N}^{-N_{4}}$ & $\mathrm{~N}^{-\mathrm{NO}_{2}}$ & $\mathrm{~N}^{-\mathrm{NO}_{3}}$ & ${\mathrm{P}-\mathrm{PO}_{4}}$ \\
\hline $\mathrm{S}$ & $-1,0$ & 0,9 & $-0,9$ & $-0,9$ & $-1,0$ & $-0,96$ & $-0,9$ & $-0,98$ & $-0,9$ \\
$\mathrm{H}$ & $\underline{-1,0}$ & 1,0 & $-1,0$ & $-1,0$ & $-1,0$ & $-0,99$ & $-1,0$ & $\underline{-1,00}$ & $-1,0$ \\
$\mathrm{Eq}$ & $\underline{-1,0}$ & 0,9 & $-1,0$ & $-1,0$ & $-1,0$ & $-0,98$ & $-1,0$ & $-0,99$ & $-1,0$ \\
$\mathrm{D}$ & 1,0 & $\underline{-1,0}$ & $\underline{1,0}$ & $\underline{1,0}$ & 1,0 & 0,99 & 1,0 & 0,97 & $\underline{1,0}$ \\
$\mathrm{IB}$ & $-1,0$ & $\underline{1,0}$ & $\underline{-1,0}$ & $\underline{-1,0}$ & $-1,0$ & $\underline{-1,00}$ & $\underline{-1,0}$ & $-0,99$ & $\underline{-1,0}$ \\
mHBI & 1,0 & $-0,8$ & 0,9 & 0,9 & 0,9 & 0,92 & 0,9 & 0,95 & 0,9 \\
BMWP & $-1,0$ & 0,9 & $-0,9$ & $-0,9$ & $-1,0$ & $-0,96$ & $-0,9$ & $-0,98$ & $-0,9$ \\
ASPT & $-1,0$ & 1,0 & $-1,0$ & $-1,0$ & $\underline{-1,0}$ & $\underline{-1,00}$ & $-1,0$ & $\underline{-1,00}$ & $-1,0$ \\
\hline
\end{tabular}

a Unités des variables illustrées dans le tableau 2

brassage de l'eau maintenant l'oxygénation à un niveau plus ou moins élevé.

Les matières oxydables, mesurées par la demande biologique en oxygène $(\mathrm{DBO})$ et la demande chimique en oxygène (DCO), sont parmi les paramètres qui ont été pris en compte pour évaluer l'incidence de la pollution organique sur le cours d'eau étudié. Les valeurs moyennes de DBO et de DCO sont enregistrées au niveau de la station du rejet principal (RP) avec respectivement $658 \mathrm{mg} \mathrm{d} \mathrm{O}_{2} \cdot \mathrm{L}^{-1}$ et $1305 \mathrm{mg} \mathrm{d} \mathrm{O}_{2} \cdot \mathrm{L}^{-1}$. Dans la station du Pont de Taourirt (PT) et d'Ouled Lefkir $(\mathrm{LF})$, les teneurs moyennes en $\mathrm{DBO}_{5}$ sont respectivement de 5,4 et $8 \mathrm{mg} \mathrm{d} \mathrm{O}_{2} \cdot \mathrm{L}^{-1}$. Les teneurs en DCO sont de 11,3 et de $18,4 \mathrm{mg} \mathrm{d} \mathrm{O}_{2} \bullet \mathrm{L}^{-1}$ pour les stations PT et LF.

Le suivi des paramètres azotés et phosphorés a montré que la station RP présente des valeurs élevées par rapport aux autres stations PT et LF (Tableau 2). Ceci s'explique par l'apport excessif de ces polluants par les rejets des eaux usées au niveau de cette station.

Les résultats des différentes analyses de macroinvertébrés des stations étudiées sont présentés dans l'annexe A). Ils rapportent que l'abondance moyenne par mètre carré (Individus $\bullet \mathrm{m}^{-2}$ ) est de l'ordre de 28686 dans la station PT et de 69933 dans la station LF. En RP, l'abondance est importante, 48420 malgré une faible richesse. Ceci est dû à la prolifération des taxons résistants à la pollution surtout les Diptères et les Oligochètes. En ce qui concerne la richesse taxonomique (S), elle est respectivement de l'ordre de 52; 16 et 38 taxons pour PT, RP et LF. Quant à l'indice diversité de Shannon-Wiener $(\mathrm{H})$, il est de l'ordre de 3,74, 0,69 et 3,02 respectivement pour PT, RP et LF. Pour l'équitablité (Eq) des différentes stations, elle est de
84,06 pour PT, 14,73 pour RP et de 62,41 pour LF. Par contre, l'indice de Simpson (D) varie entre 0,099 pour PT, 0,83 pour $\mathrm{RP}$ et 0,052 pour la station LF. L'indice biotique (IB) quant à lui présente des valeurs élevées pour les stations PT et LF avoisinant 9 et témoignant ainsi d'une eau de bonne qualité. Par contre, au niveau de la station recevant le rejet d'eau usée $\mathrm{RP}$, son indice est de 2,6 indiquant que les eaux de cette station sont de mauvaise qualité. De même, le BMWP (Biological Monitoring Working Party) et l'ASPT (Average Score Per Taxa) sont faibles au niveau de la station RP et présentent des valeurs élevées dans les autres stations (PT et LF), alors que l'indice de mHBI (Modified Hilsenhoff Biotic Index) présente une valeur élevée au niveau de la station RP (station polluée). Les valeurs enregistrées au niveau de la station recevant le rejet des eaux usées (RP) sont dues à la disparition des taxons sensibles à la pollution tel le cas des Éphéméroptères, Trichoptères et Odonates et à la prolifération des taxons tolérants à la pollution organique, notamment les Tubificidae et les Chironomini (ETO, Tableau 2 et Annexe A).

Le tableau 3 résume les corrélations entre les variables chimiques et les indices biotiques. De même, le tableau 4 présente les corrélations entre les différents variables biologiques.

\section{DISCUSSION}

$\mathrm{Du}$ point de vue biogéographique, la macrofaune du secteur étudié appartient à la zone paléarctique avec des espèces endémiques marocaines ou maghrébines, notamment Ecdyonurus rothschildi, Hydropsyche maroccana, Hydropsyche resminida (BERRAHOU et al., 2001a). Le peuplement en 


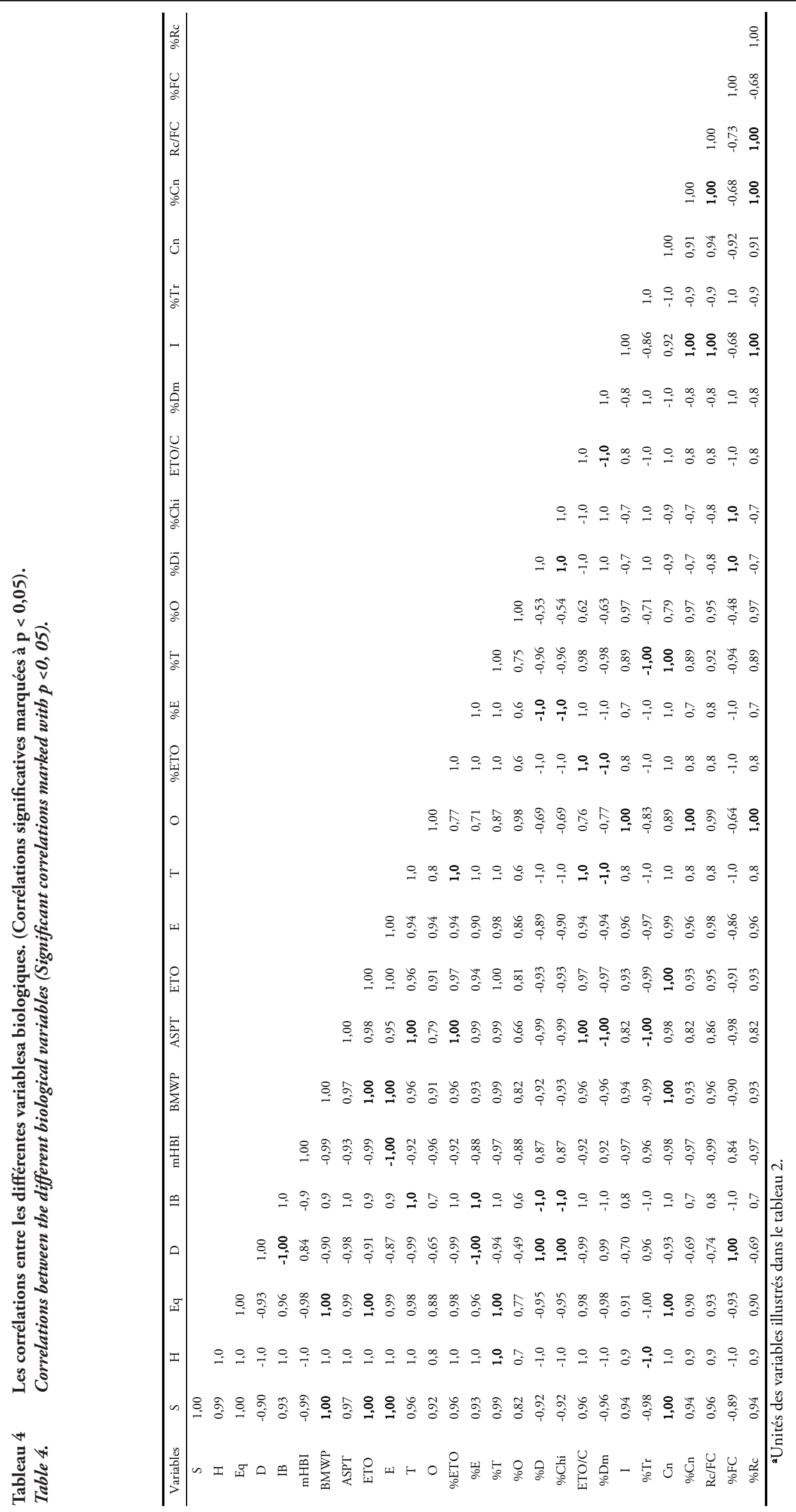


macroinvertébrés benthiques récoltés montre l'absence totale de Plécoptères. Ainsi, de nombreux travaux (DAKKI, 1986; CHERGUI et al., 1990; GIUDICELLI et DAKKI, 1984; GIUDICELLI et al., 1985) mentionnent que les Plécoptères se limitent aux seules eaux courantes bien oxygénées de hautes altitudes. La rareté ou même l'absence des Plécoptères dans certains cours d'eau d'Afrique du Nord semble être due aux températures estivales élevées (BERRAHOU et al., 2001a). Le secteur étudié se distingue par la présence des Éphéméroptères, des Coléoptères et les Trichoptères thermophiles, voire potamiques (Caenis luctuosa, Baetis pavidus, Hydropsyche maroccana) qui caractérisent les rivières marocaines à températures estivales élevées (DAKKI 1986).

BOYLE et FRALEIGH (2003) etVOELZ et MAcARTHUR (2000) ont montré que la distribution des macroinvertébrés est influencée par un grand nombre de facteurs de l'environnement telles la disponibilité d'aliments (PEETERS et al., 2004), les conditions hydrauliques (VOELZ et al., 2000), la composition du substrat (SANDIN et JOHNSON, 2004), l'augmentation de la charges nutritive (BUSS et al., 2002; CAMARGO et al., 2004) et la compétition et la prédation (PIANKA, 1983; FEMINELLA et RESH, 1990). De plus, certains auteurs ont indiqué que la faune invertébrée de différents habitats répond différemment aux variations de la qualité de l'eau (ILIOPOULOU-GEORGUDAKI et al., 2003; PARSONS et NORRIS, 1996; SOLDNER et al., 2004). L'interaction de ces facteurs détermine quelques gradients dans la richesse d'invertébrés. Dans un cours d'eau naturel, la richesse augmente de l'amont vers l'aval, comme le cas du Zegzel au Maroc Oriental (BERRAHOU, 1988). Par contre, dans l'oued $\mathrm{Za}$, la richesse minimale est observée au niveau de la station du rejet. Selon WARD et STANFORD (1979), le rejet a entraîné un discontinuum du gradient amont aval du cours d'eau.

De même, AZRINA et al. (2006), CEREGHINO et al. (2002), MINSHALL et al. (1985), RESH et JACKSON (1993) et VANNOTE et al. (1980) ont observé que la richesse spécifique est sensible à l'impact des activités humaines sur les écosystèmes aquatiques, en particulier sur les insectes aquatiques qui sont souvent de bons indicateurs de conditions environnementales dans les cours d'eau, donc très sensibles à la pollution. La richesse peut être un bon descripteur de l'influence de perturbations anthropiques sur les ruisseaux (COLES et al., 2004; COMPIN et CEREGHINO, 2003).

Les paramètres physico-chimiques classiques, les indices de diversité (SHANNON et WEAVER, 1949; SIMPSON, 1949), les indices biotique (TUFFERY et VERNEAUX, 1968) et les autre indices "Modified Hilsenhoff Biotic Index " (BARBOUR et al., 1999), "Biological Monotoring Working Party " (ARMITAGE et al., 1983) et " Average Score Per Taxa " (FRIEDRICH et al.,1996) montrent que la qualité des stations étudiées, PT, RP et LF, est respectivement bonne, polluée et assez bonne. La station témoin PT est diversifiée et contient des taxons considérés sensibles à la pollution et qui appartiennent aux grands groupes des Trichoptères, des Éphéméroptères et des Odonates (GERRITSEN et al., 1998). Dans la station polluée RP, le peuplement est peu diversifié et déséquilibré. La forte pollution a entrainé la disparition des taxons sensibles à la pollution et la prolifération des taxons résistants à la pollution dont la présence est liée à l'abondance de la matière organique des eaux usées. Il s'agit principalement des Oligochètes, des Tubificidae et des Diptères Chironomini. DUMNICKA (2002) et GRZYBKOWSKA (1993) ont montré d'une façon significative l'augmentation de densité des Oligochètes et des Chironomidae dans les rivières affectées par la pollution organique. Les Chironomidae sont plus tolérants à la pollution et peuvent avoir des mécanismes de recolonisation plus efficaces (PIRES et al., 2000). De même, GIUDICELLI et al. (1981) ont montré que la pollution se traduit principalement par une augmentation de la densité des communautés des habitats lénitiques (Diptères et Oligochètes) où se dépose la matière organique. Vers l'aval LF, la qualité s'est améliorée grâce à l'autoépuration, qui consiste en la transformation de matière organique en matière minérale grâce aux microorganismes. Ainsi, il y a réapparition de la faune des eaux propres observée dans la station de référence PT (les Ecdyonuridae, les Hydropsychidae, les Caenidae et les Baetidae). Cependant, on constate un certain déséquilibre par rapport à celle-ci, dû à la forte abondance des Tubificidae, des Baetidae, des Hydropsychidae et des Chironomidae. Cette forte densité est liée à l'abondance de la nourriture, notamment les algues et les fins débris organiques.

\section{CONCLUSION}

Le cours inférieur de l'oued Za est très dégradé au niveau du rejet des eaux usées en aval de la ville de Taourirt. Il est nécessaire de le protéger par la canalisation puis l'épuration de ce rejet avant son déversement dans le milieu récepteur.

\section{RÉFÉRENCES BIBLIOGRAPHIQUES}

AFNOR, Association française de Normalisation. (1986). Eaux

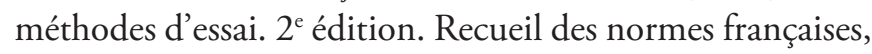
Paris, France.

AFNOR, Association française de Normalisation. (1994). Recueil des normes françaises : qualité de l'eau. Environnement, Paris, France. 
ARMITAGE, P.D., D. MOSS, J.T. WRIGHT et M.T. FURSE (1983). The performance of the new biological water quality score system based on macroinvertebrates over a wide range of unpolluted running water sites. Water Res., $17,333-347$.

AZRINA, M.Z., C.K. YAP, A. RAHIM ISMAIL, A. ISMAIL et S.G. TAN (2006). Anthropogenic impacts on the distribution and biodiversity of benthic macroinvertebrates and water quality of the Langat River, Peninsular Malaysia. Ecotox. Environ. Saf., 64, 337-347

BARBOUR, M.T., J. GERRITSEN, G.E. GRIFFITH, R. FRYDENBORG, E. MCCARRON, J.S. WHITE et M.L. BASTIAN (1996). A framework for biological criteria for Florida streams using benthic macroinvertebrates. J. North Am. Benthol. Soc., 15, 185-211.

BARBOUR, M.T., J. GERRITSEN, B.D. SNYDER et J.B. STRIBLING (1999). Rapid bioassessment protocols for use in wadeable rivers: Periphyton, benthic macroinvertebrates, and fish. $2^{\mathrm{e}}$ édition, EPA 841-B-99-002. US Environmental Protection Agency, Office of Water, Washington, DC.

BARBOUR, M.T., J.B. STRIBLING et J.R. KARR (1995). Multimetric approach for establishing biocriteria and measuring biological condition. Dans : Biological Assessment and Criteria. Tools for Water Resource Planning and Decision Making. Lewis Publishers, Boca Raton, FL, USA, pp. 63-77.

BERRAHOU, A. (1988). Recherches hydrobiologiques sur un cours d'eau des Beni-Snassene (Maroc Nord-Oriental). Impact des crues sur les macroinvertébrés benthiques. Thèse $3^{\mathrm{e}}$ cycle, Univ. Rabat, Maroc, 124 p.

BERRAHOU, A., B. CELLOT et P. RICHOUX (2001a). Distribution longitudinale des macroinvertébrés benthiques de la Moulouya et de ces principaux affluents (Maroc). Ann. Limnol., 37, 223-235.

BERRAHOU, A., G. CHAVANAN et P. RICHOUX (2000). Études sur la basse Moulouya (Maroc oriental) : 6- Les Coléoptères aquatiques de l'oued Zegzel. Bull. Mens. Soc. Limn. Lyon. 69, 44-50.

BERRAHOU, A., G. CHAVANON, A. BELLOUALI et P. RICHOUX (2001b). Études sur la basse Moulouya (Maroc oriental) : 7- Les Coléoptères aquatiques de l'oued Za. Bull. Mens. Soc. Linn, 70, 127-131.
BODE, R.W., M.A. NOVAK, L.E. ABELE, D.L. HEITZMAN et A.J. SMITH (2002). Quality assurance work plan for biological stream monitoring in New York State. Stream Biomonitoring Unit, Bureau of Water Assessment and Management, Division of Water, NYS Department of Environmental Conservation, Albany, NY, USA, 41p.

BOYLE, T.P. et H.D. FRALEIGH JR. (2003). Natural and anthropogenic factors affecting the structure of the benthic macroinvertebrate community in an effluent-dominated reach of the Santa Cruz River, AZ, USA. Ecol. Ind., 3, 93-117.

BUFFAGNI, A., G. CROSA, D.M. HARPER etJ.L. KEMP (2000). Using macroinvertebrate species assemblages to identify river channel habitat units: an application of the functional habitat concept to a large, unpolluted Italian river (River Ticino, Northern Italy). Hydrobiologia, 435, 213-225.

BUSS, D.F., D.F. BAPTISTA, M.P. SILVEIRA, J.L. NESSIMIAN et L.F. DORVILLE (2002). Influence of water chemistry and environmental degradation on macroinvertebrate assemblages in a river basin in SouthEast Brazil. Hydrobiologia, 481, 125-136.

BUSTOS-BAEZ, S. et C.C. FRID (2003). Using indicator species to assess the state of macrobenthic communities. Hydrobiologia,: 496, 299-09.

CAMARGO, J.A., A. ALONSO et M. DE LA PUENTE (2004). Multimetric assessment of nutrient enrichment in impounded rivers based on benthic macroinvertebrates. Environ. Monitor. Ass., 96, 233-249.

CEREGHINO, R., P. CUGNY, P. et P. LAVANDIER (2002). Influence of intermittent hydropeaking on the longitudinal zonation patterns of benthic invertebrates in a mountain stream. Int. Rev. Hydrobiol., 87, 47-60.

CHERGUI, H., G. CHAVANON, A. BERRAHOU et M. MELHAOUI (1990). À propos des Plécoptères du Maroc oriental. Bull. Inst. Sci. Rabat., 14, 51-53.

COLES, J.F., T.F. CUFFNEY, G. McMAHON et K.M. BEAULIEU (2004). The effects of urbanization on the biological, physical, and chemical characteristics of coastal New England streams. U.S. Geological Survey Professional Paper 1695, 47p.

COMPIN, A. et R. CEREGHINO (2003). Sensitivity of aquatic insect species richness to disturbance in the AdourGaronne stream system (France). Ecol. Indic., 3, 135-142. 
DAKKI, M. (1986). Recherches hydrobiologiques sur le Haut Sebou (Moyen-Atlas). Une contribution à la connaissance faunistique, écologique et historique des eaux courantes sudméditerranéennes. Thèse d'État, Univ. Mohamed V, Fac. Rabat, 214 p.

DE PAUW, N. et G. VANHOOREN (1983). Method for biological water quality assessment of watercourses in Belgium. Hydrobiologia, 100, 153-184.

DUMNICKA, E. (2002). Upper Vistula river: response of aquatic communities to pollution and impoundment. X. Oligochaete taxocens. Polish J. Ecol., 50, 237-247.

FEMINELLA, J.W. et V.H. RESH (1990). Hydrologic influences, disturbance, and intraspecific competition in a stream caddisfly population. Ecology, 71, 2083-2094.

FRIEDRICH, G., D. CHAPMAN et A. BEIM (1996). The use of biological material in water quality assessments: A guide to the use of biota, sediments and water in environmental monitoring. $2^{\mathrm{e}}$ édition, D. CHAPMAN (Éditeurs), New York, NY, USA.

GERRITSEN, J., R.E. CARLSON, D.L. DYCUS, C. FAULKNER, G.R. GIBSON, J. HARCUM et S.A. MARKOWITZ (1998). Lake and reservoir bioassessment and biocriteria. Technical guidance document. US Environmental Protection Agency. EPA 841-B-98-007.

GIUDICELLI, J. et M. DAKKI. (1984). Les sources du Moyen Atlas et du Rif (Maroc) : faunistique (Description de deux espèces nouvelles de trichoptères), écologie, intérêt biogéographique. Bijdragen tot die Dierkunde, 54, 83-100.

GIUDICELLI, J., M. DAKKI et A. DIA (1985). Caractéristiques abiotiques et hydrobiologiques des eaux courantes méditérranéennes. Ver. Internat.Verein Limnol.,22, 2094-2101.

GIUDICELLI, J., P. LEGIER, J.J. MUSSO et G. PREVOT (1981). Impacts des pollutions organiques sur deux cours d'eau de la région méditerranéenne. Recherches sur la validité des méthodes biologiques d'évaluation de la qualité des eaux courantes en région méditerranéenne. Univ. AixMarseille III, Labo. Biol. Anim. (écologie), Ministère de l'Environnement (Comité Eau) contrat no 78-128, 96 p.

GRZYBKOWSKA, M. (1993). Chironomidae w bentosie i dryfie odcinków rzek o róźnej rz edowos'ci w Polsce Środkowej. Łódz' : Acta Univ. Lodz, 119 p.
HELLAWELL J.M. (1986). Biological indicators of freshwater pollution and environmental management. Elsevier (Éditeur), Londres, Grande-Bretagne, 546 p.

HILSENHOFF, W.L. (1988). Rapid field assessment of organic pollution with a family-level biotic index. J. North Am. Benthol. Soc., 7, 65-68.

ILIOPOULOU-GEORGUDAKI，J., V. KANTZARIS，P. KATHARIOS, P. KASPIRIS, TH. GEORGIADIS et B. MONTESANTOU (2003). An application of different bioindicators for assessing water quality: a case study in the rivers Alfeios and Pineios (Peloponnisos, Greece). Ecol. Indic., 2, 345-360.

LORENZ, A., D. HERING, C.K. FELD et P. ROLAUFFS (2004). A new method for assessing the impact of hydromorphological degradation on the macroinvertebrate fauna of five German stream types. Hydrobiologia, 516, $107-127$.

MANDAVILLE, S.M. (2002). Benthic macroinvertebrates in freshwaters-taxa tolerance values, metrics and protocols. Professional Lake Manage, (Project H-1) Soil and Water Conservation Society of Metro Halifax, 48 p.

MINSHALL, G.W., R.C. PETERSEN et C.F. NIMZ (1985). Species richness in streams of different size from the same drainage basin. Am. Naturalist, 125, 16-38.

NEDEAU, E.J., R.W. MERRITT et M.G. KAUFMAN (2003). The effect of an industrial effluent on an urban stream benthic community: water quality vs. habitat quality. Environ. Pollut., 123, 1-13.

NORRIS R.H. et A. GEORGES (1993). Analysis and interpretation of benthic macroinvertebrate surveys. Dans : Freshwater Biomonitoring and Benthic Macro-invertebrates. ROSENBERG D.M. et V.H. RESH (Éditeurs), pp. 234286. Chapman and Hall, New York, NY, USA.

PARSONS, M. et R.H. NORRIS (1996). The effect of habitatspecific sampling on biological assessment of water quality using a predictive model. Freshwater Biol., 36, 419-434.

PEETERS, E.T.H., R. GYLSTRA et J.H. VOS (2004). Benthic macroinvertebrate community structure in relation to food and environmental variables. Hydrobiologia, 519, 103-115.

PIANKA, E.R. (1983). Evolutionary ecology, (3 $3^{\text {rd }}$ ed.) Harper and Row, New York, NY, USA, 416 p. 
PIRES, A.M., I.G. COWX et M. COELHO (2000). A benthic macroinvertebrate community of intermittent streams in the middle reaches of the Guadiana Basin (Portugal). Hydrobiologia, 435, 167-175.

RESH, V.H. et J.K. JACKSON (1993). Rapid assessment approaches to biomonitoring using benthic macroinvertebrates. Dans : Freshwater Biomonitoring and Benthic Macroinvertebrates. ROSENBERG, D.M. et V.H. RESH (Éditeurs). Chapman and Hall, Londres, GrandeBretagne, pp. 195-223.

RESH, V.H., R.H. NORRIS et M.T. BARBOUR (1995). Design and implementation of rapid assessment approaches for water resource monitoring using benthic macroinvertebrates, Australian J. Ecol., 20, 108-121.

REYNOLDSON, T.B., R.C. BAILEY, K.E. DAY et R.H. NORRIS (1995). Biological guidelines for freshwater sediment based on BEnthic Assessment of SedimenT (BEAST) using a multivariate approach for predicting biological state. Australian J. Ecol., 20, 198-219.

SANDIN, L. et R.K. JOHNSON (2004). Local, landscape and regional factors structuring benthic macroinvertebrate assemblages in Swedish streams. Landscape Ecol., 19, 501-514.

SHANNON, C. E. et W. WEAVER (1949). The mathematical theory of communication. University of Illinois Press, Urbana, IL, USA, 117 p.

SIMPSON, E.H. (1949). Measurement of diversity. Nature, $163,688-688$.

SOLDNER, M., I. STEPHEN, L. RAMOS, R. ANGUS, N. CLAIRE WELLS, A. GROSSO et M. CRANE (2004). Relationship between macroinvertebrate fauna and environmental variables in small streams of the Dominican Republic. Water Res., 38, 863-874.

SOLIMINI, A.G., M. BAZZANTI, A. RUGGIERO et G. CARCHINI (2008). Developing a multimetric index of ecological integrity based on macroinvertebrates of mountain ponds in central Italy. Hydrobiologia, 597, 109-123.

THORNE, R.S. T.J. et W.P. WILLIAMS (1997). The response of benthic macroinvertebrates to pollution in developing countries: a multimetric system of bioassessment. Freshwater Biol., 37, 671-686.
TUFFERY, G. et J. VERNEAUX (1968). Méthode de détermination de la qualité biologique des eaux courantes. Trav. Sect. Tech. et Pisc., CERAFER, Paris, France, 23 p.

USEPA (2000). A stream condition index for West Virginia wadeable streams. Contract EPA-68-C7-0014. to Tetra Tech, Inc. Environmental Services Division, Office of Science and Technology, Office of Water , 24 p.

USEPA (1998). Lake and reservoir bioassessment and biocriteria: Technical guidance document. Washington, DC, EPA 841B-98-007. Office of Wetlands, Oceans, and Watersheds (4503F), Office of Science and Technology (4304), Office of Water, United States Environmental Protection Agency.

USEPA (2002). Methods for evaluating wetland c ondition: Developing metrics and indexes of biological integrity. Washington, DC. EPA-822-R-02-016. Office of Water, United States Environmental Protection Agency, 38 p.

VANNOTE, R.L., G.W. MINSHALL, K.W. CUMMINS, J.R. SEDELL et C.E. CUSHING (1980). The river continuum concept. Can. J. Fish. Aquat. Sci., 37, 130-137.

VOELZ, N.J. et J.V. MAcARTHUR (2000). An exploration of factors influencing lotic insect species richness. Biodivers. Conserv., 9, 1543-1570.

VOELZ, N.J., S. SHIEN et J.V. WARD (2000). Long-term monitoring of benthic macroinvertebrate community structure: A perspective from a Colorado river. Aquat. Ecol., 34, 261-278.

WARD, J.V. et J.A. STANFORD (1979). Ecological factors controlling stream zoobenthos with emphasis on thermal modification of regulated streams. Dans : The Ecology of Regulated Streams. WARD, J.V. et J.A. STANFORD (Éditeurs). Plenum Press, New York, NY, USA, pp. 35-55.

WRIGHT, J.F., M.T. FURSE et P.D. ARMITAGE (1993). RIVPACS: A technique for evaluating the biological quality of rivers in the UK. European Water Pollut. Control., 3, 15-25. 
ANNEXE A : Le tableau d'abondance totale moyenne $\left(\mathrm{N} \cdot \mathrm{m}^{-2}\right)$ de six prélèvements d'échantillonnage durant la période d'étude (juin 2004-novembre2004).

APPENDIX A: Average density macroinvertebrates $\left(\mathrm{N} \cdot \mathrm{m}^{-2}\right)$ from six sampling campaigns during the study period (June 2004-November 2004). PT: Bridge of Taourirt; RP: Principal Discharge; LF: Ouled Lefkir.

\begin{tabular}{|c|c|c|c|}
\hline Stations & PT & $\mathrm{RP}$ & LF \\
\hline Plathelminthes & 633,3 & 0 & 0 \\
\hline Dugesia gonocephala & 633,3 & 0 & 0 \\
\hline Annélides & 66,6 & 580 & 7540 \\
\hline Naididae & 0 & 0 & 6,6 \\
\hline Tubificidae & 20 & 553,3 & 6813,3 \\
\hline Helobdella sp & 46,6 & 26,6 & 720 \\
\hline Mollusques & 166,6 & 0 & 406,6 \\
\hline Melanopsis cariosus & 6,6 & 0 & 0 \\
\hline Melanopsis costellata & 13,3 & 0 & 0 \\
\hline Melanopsis premorsa & 53,3 & 0 & 0 \\
\hline Melanopsis sp & 26,6 & 0 & 0 \\
\hline Physa acuta & 66,6 & 0 & 406,6 \\
\hline Crustacées & 13,3 & 0 & 6,6 \\
\hline Asellus sp & 13,3 & 0 & 0 \\
\hline Gammarus sp & 0 & 0 & 6,6 \\
\hline Ephéméroptères & 14313 & 0 & 34206,6 \\
\hline Baetis neglectus & 4706,6 & 0 & 21500 \\
\hline Baetis pavidus & 246,6 & 0 & 1480 \\
\hline Baetis rhodania & 386,6 & 0 & 33,3 \\
\hline Caenis luctuosa & 7580 & 0 & 11180 \\
\hline Choroterpes $s p$ & 133,3 & 0 & 0 \\
\hline Cloeon sp & 66,6 & 0 & 0 \\
\hline Ecdyonurus rothschildi & 326,6 & 0 & 13,3 \\
\hline Oligoneuriella skhounate & 733,3 & 0 & 0 \\
\hline Leptophlebia sp & 133,3 & 0 & 0 \\
\hline Odonates & 153,3 & 0 & 20 \\
\hline Aeschnidae & 13,3 & 0 & 0 \\
\hline Corduliidae & 13,3 & 0 & 0 \\
\hline Calopterygidae & 13,3 & 0 & 0 \\
\hline Gomphidae & 113,3 & 0 & 20 \\
\hline Hétéroptères & 173,3 & 33,3 & 2913,3 \\
\hline Corixa sp & 6,6 & 0 & 0 \\
\hline Micronecta & 166,6 & 33,3 & 2913,3 \\
\hline Coléoptères & 173,3 & 33,3 & 100 \\
\hline Dryops sp & 0 & 0 & 13,3 \\
\hline Hydroporus & 33,3 & 0 & 13,3 \\
\hline Hygrobidae & 13,3 & 0 & 0 \\
\hline Laccobius & 13,3 & 6,6 & 46,6 \\
\hline Laccophilus & 6,6 & 13,3 & 0 \\
\hline Limnius sp & 66,6 & 0 & 0 \\
\hline Normandia & 6,6 & 0 & 0 \\
\hline Potamonectes sp & 13,3 & 13,3 & 13,3 \\
\hline Yola sp & 20 & 0 & 13,3 \\
\hline
\end{tabular}




\begin{tabular}{|c|c|c|c|}
\hline Stations & PT & $\mathrm{RP}$ & LF \\
\hline Trichoptère & 9046,6 & 0 & 15746,6 \\
\hline Agraylea sp & 486,6 & 0 & 6,6 \\
\hline Hydropsyche maroccana & 3313,3 & 0 & 13400 \\
\hline Hydropsyche pellucidula & 1960 & 0 & 940 \\
\hline Hydropsyche resmineda & 1893,3 & 0 & 166,6 \\
\hline Hydropsyche sp & 400 & 0 & 526,6 \\
\hline Hydroptila & 580 & 0 & 333,3 \\
\hline Philopotamidae & 286,6 & 0 & 340 \\
\hline Polycentropodidae & 113,3 & 0 & 33,3 \\
\hline Psychomyidae & 13,3 & 0 & 0 \\
\hline Diptères & 3939,7 & 50512,8 & 8986,3 \\
\hline Anthomyidae & 100 & 26,6 & 40 \\
\hline Culicidae & 20 & 0 & 60 \\
\hline Certopogonidae & 6,6 & 0 & 0 \\
\hline Chironomini & 1746,6 & 6213,3 & 5886,6 \\
\hline Cylindromyidae & 0 & 0 & 6,6 \\
\hline Empididae & 6,6 & 0 & 0 \\
\hline Ephydridae & 0 & 0 & 20 \\
\hline Glyptotendipes & 0 & 43820 & 313,3 \\
\hline Orthocladinae-Diamesinae & 493,3 & 0 & 140 \\
\hline Psychodidae & 0 & 246,6 & 0 \\
\hline Ptychopteridae & 0 & 0 & 6,6 \\
\hline Rhagionidae & 0 & 6,6 & 0 \\
\hline Athericidae & 0 & 6,6 & 6,6 \\
\hline Simulidae & 513,3 & 0 & 1280 \\
\hline Stratiomyidae & 0 & 6,6 & 0 \\
\hline Syrphidae & 0 & 13,3 & 0 \\
\hline Tabanidae & 66,6 & 0 & 60 \\
\hline Tanypodinae & 533,3 & 0 & 626,6 \\
\hline Tanytarsini & 360 & 146,6 & 546,6 \\
\hline Tipulidae & 100 & 26,6 & 0 \\
\hline
\end{tabular}

